\title{
Effect of Endophytic Consortium on Pisum sativum and Vigna angularis Plant Analyst with FTIR Spectroscopy
}

\section{Rathod Zalak R and Saraf Meenu S*}

Department of Microbiology and Biotechnology, University School of Sciences, Gujarat University, Ahmedabad, Gujarat, India

*Corresponding Author: Saraf Meenu S, Department of Microbiology and Biotechnology, University School of Sciences, Gujarat University, Ahmedabad, Gujarat, India.
Received: October 21, 2021

Published: November 24, 2021

(C) All rights are reserved by Rathod Zalak $\mathbf{R}$ and Saraf Meenu S.

\begin{abstract}
Endophytes have a property of biofertilizer as well as they work as PGPB and good source of secondary metabolites as phytochemicals properties too. The object of this study was to identify functional groups present in plant extract as phytochemicals for plant of Pisum sativum and Vigna angularis which were inoculated with consortium of Bacillus subtilis, Bacillus tequilensis, Bacillus amyloliquefaciens and test plant of Pisum sativum and Vigna angularis which are inoculated with consortium of Bacillus subtilis, Bacillus tequilensis, Bacillus amyloliquefaciens. With compared of control remains as uninoculated with endophytes. Here we can distinguish changes in functional group of test plant and control plant of Pisum sativum and Vigna angularis and differentiate them through FTIR of plant extract due to the presence of endophytic consortium of three different bacteria.
\end{abstract}

Keywords: Endophytic consortium; FTIR; Pisum sativum; Vigna angularis

\section{Introduction}

FTIR (Fourier Transform Infrared Spectroscopy) is vibrational spectroscopic method which is sample, rapid, sensitive, accurate [13] and non-destructive to tissue and only small amount of material with minimum sample preparation are required. This type of analysis can be used for characterizing samples in forms of liquids, solutions, pastes powders, films, fibres and glasses. The analysis is also possible for analysing material on the surface of substrate [10]. These techniques also require molecular level information allowing investigation of functional groups [17], bonding types and molecular confirmation. Spectral bands in vibrational spectra are molecule specific and provide direct information about biochemical composition. These bands are relatively narrow, easy to resolve, and sensitive to molecular structure, confirmation and environment [21].

Pea, (Pisum sativum), also known as garden pea, herbaceous annual plant in the family Fabaceae, grown virtually worldwide for its edible seeds. Peas can be bought fresh, canned or frozen and dried peas are commonly used in soups. Some varieties, including sugar peas and snow peas, produce pods that are edible and are eaten raw or cooked like green beans; that are popular in East Asian cuisines. The plants are fairly easy to grow, and the seeds are a good source of protein and dietary fibre (Britannica). Pea plants exhibit an indeterminate growth habit. The first nodes, some of which give rise to branches, are vegetative, while subsequent nodes are reproductive node. The number of seeds per nod depends on the variety and the environmental conditions. There is a large genetic variability for number of branches, pods, seeds per pod, thousandseed weight, leaf area, height of the plant. However, when crosses are made which attempt to improve on, and combine these traits, yields are often reduced [5]. When vegetative growth is vigorous, there is increased interplant competition and yield suffers. Reduction in leaf area and plant height to produce smaller, more highly branched plants and increasing of the thousand seed weight favours yield [4] Starch is the main component of the pea seed, but occurs in several forms. Smooth-seeded varieties have round starch granules, whereas most of those varieties with wrinkled-seed have composite granules. Every group has a clearly defined starch content as well as amylopectin and amylose composition [6]. Varia- 
tions in the synthesis of starch, with smooth-seeded group having a higher starch and amylopectin content than wrinkled seeded types results in genetic variability in protein content [4]. The total soluble sugar in smooth seeds seems to be much lower than that of wrinkled-seed which verbascose and stachyose in higher amounts [6]. They exhibit relatively low trypsin inhibitor activity $[7,18]$. Their yield is highly affected by environmental stresses caused by cold and drought. Other than that, diseases caused by certain organisms also reduce the yield. Diseases caused by fungi include Ascochyta blight, downy mildew, powdery mildew, fusarium wilt. Diseases caused by viruses include pea common mosaic, pea enation mosaic, pea top yellow, pea seed-borne mosaic, etc. Disease caused by bacteria is bacterial blight [5]. Theadzuki bean (Vignaangularis) has been cultivated and used for many centuries in the Orient where it is a popular ingredient for many types of-sweetened desserts. The adzuki bean contains about $50 \%$ starch and $20-25 \%$ protein [11,19,30,35]. Although the major component of legume seeds in general is starch, greater attention has been given to their proteins [36]. The research showed that the total starch in adzuki beans was $43.2 \%$ [36]. Other researchers have reported wide variations which have been attributed mainly to differences in isolation methods [22]. When starch is heated with excess water, the starch granule swells and its volume increase which is known as swelling power [8]. The gelatinization property is closely related to the pasting property of starch (Ai and Jane, 2015). The gelatinization parameters of the adzuki bean starches were intermediate among those of legume starches [37]. Several reports have associated the swelling power with gelatinization and pasting properties of starch [1,34]. The apparent amylose content was strongly positively related to the swelling, gelatinization, and pasting properties of starch, because these properties depend on the amylopectin content $[12,16,31,32]$. According to a report the phosphorous in adzuki bean amylopectin existed as phosphate monoester (Yoshimoto., et al. 2001). The distributions of the branched chain lengths of adzuki bean starches were analysed by HPIC. The chain content in the adzuki bean was higher other beans [12]. The branch chain distribution of the amylopectin component also affected the gelatinization and pasting properties of starch [14].

\section{Materials and Methods}

\section{Isolation and characterization}

Isolation of organisms was done from leaf of Citrus limon (Lemon) tree. Sampling was done from Department of Horticulture,
Anand Agriculture University, Anand, Gujarat, India. $22.5359^{\circ} \mathrm{N}$, $72.9749^{\circ}$ E. Accordingly, isolates are endophytes in nature. Three isolates are obtained and labelled as isolate $\mathrm{D}$, isolate $\mathrm{N}$, and isolate A. Characterization of these isolates were done by morphological and cultural characterization. Morphological was observed by microscopic examination through Gram staining technique given by Hans Christian Gram in (1884) with Gram staining reagents like Crystal violet, Gram's iodine, Decolourizer (Acetone), and Safranin. Cultural characteristics was noted by eye visualization of colonies grown on Nutrient agar media [28].

\section{Development of consortium}

Consortium development was attained by growing all three isolates (streak as a line) on Nutrient agar plate at $28 \pm 2{ }^{\circ} \mathrm{C}$ for 24 h. These significant developments have been made as they overlapped each other to display if they inhibit each other or not [26].

\section{Pot experimentation}

21days pot study designed on the basis of selected three endophytes spp. 10 different types of pots arranged with each bacterium and with its combination to each other to make consortium also. Total 30 pots study had been done each pot containing in triplicates of both the plants Pisum sativum and Vigna angularis. These two plants were inoculated as biofertilizer, all three isolates and consortium of these isolates once in a week. Watering was done three times in a day. Biometric results were remarked. Proline, total polyphenol, chlorophyll and gibberellic acid content was patterned after pot study. All pots study kept it in thrice [27].

\section{Extraction of plant leaves}

Plant leaves were cut first in small pieces and then crushed using a chilled mortar and pestle in chilled acetone. Ratio of leaves and chilled acetone was taken 1:10. Mixture was filtered using Whatman filter paper No. 1 and stored in Secure cap vials at $4^{\circ} \mathrm{C}$ for further study.

\section{FTIR of plant extract}

FTIR study was carried out to characterize the functional groups present in plant extract and which functional groups were located in plants with inoculation and without inoculation. FTIR was run in our laboratory (Laboratory of Department of Microbiology and Biotechnology, School of Sciences, Gujarat University, Ahmedabad) by Fourier Transform Infrared Spectrophotometer of OPUS company. Control and Test of Pisum sativum and Vigna angularis was tested by FTIR analysis. 


\section{Results and Discussion}

\section{Isolation and characterization}

Isolates were procured from leaf of Citrus limon (Lemon) tree. The isolates acquired were endophytes and they were identified as Bacillus subtilis, Bacillus tequilensis and Bacillus amyloliquefaciens based on morphological characteristics derived by Gram Staining. Bacillus subtilis had small gram positive thin rods and were arranged in chains [20]. Bacillus tequilensis had gram positive small gram positive thick rods with singular arrangement [3]. Bacillus amyloliquefaciens had small gram positive thin rods with both singular and chain arrangement [24]. This observation has been attained with the help of Experimental Microbiology Volume 1, $9^{\text {th }}$ edition [25].

\section{Development of consortium}

For the consortium development, all isolates were overlapped unto one another and tested for inhibition towards one another. The results are as shown below.

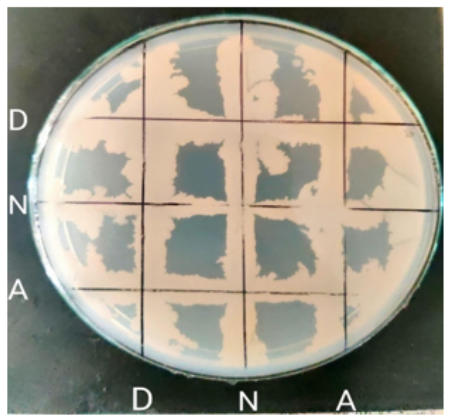

Figure 1: Consortium Development.

There have been studies of antimicrobial activity against several infections produced by a consortium of organisms. In a study, a consortium of three plant growth-promoting rhizobacteria (PGPR) strains Bacillus cereus AR156, Bacillus subtilis SM21, and Serratia sp. XY21 significantly reduced sweet pepper disease by alteration of the microbiota in rhizosphere [39]. Another example is the consortium of Bacillus velezensis MB101, Streptomyces atrovirens N23 and Trichoderma lixii NAIMCC-F-01760 was used for the management of Rhizoctonia solani that causes tomato root rot [33].

\section{Pot experimentation}

Pots of plants, Pisum sativum and Vigna angularis were studied for the influence of the isolates. The plants were observed for the enhancement in growth such as shoot length, root length, wet weight, dry weight as well as number of leaves. Other tests for both the plants were also done such as proline, total polyphenol, chlorophyll and gibberellic acid. The results are shown below:

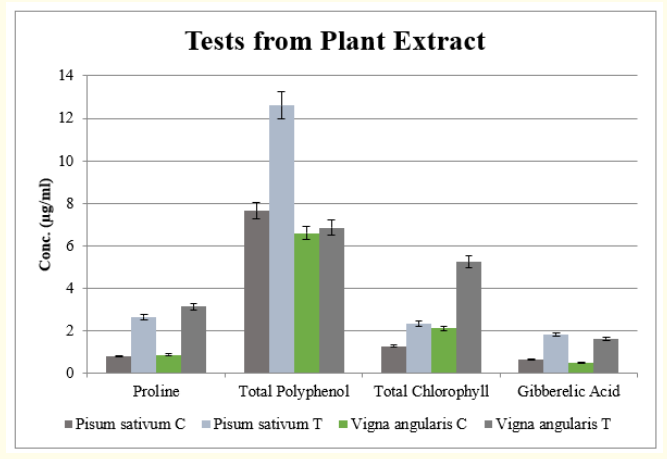

Graph 1: Effect of Consortium from Plant Extract.

Plant growth promoting microorganism inoculated macadamia plant showed higher proline content compared to untreated plants under drought condition [38]. The most effective mechanism in plants for coping against stress condition is synthesis of secondary metabolites foe example phenolic compounds [29]. Increase in phenolic compounds of Coriandrum sativum L. due to application of a Bacillus halotolerans as biofertilizer [15]. Highest chlorophyll content was observed in inoculation of PGPB. High level of Chlorophyll content could lead to higher rates of photosynthesis [2]. The concentration of gibberellic acid in the extract, on the basis of the analysis of the ethanol extract, was estimated to be $979.71 \mathrm{mg} / \mathrm{l}$ by the Dumale J.V., et al. in 2018 [9].

\section{Extraction of plant leaves}

Plant leaves were crushed with chilled acetone for further extraction. They were store in secure capped vials after filtration and stored at $4^{\circ} \mathrm{C}$ as shown in the vials below.

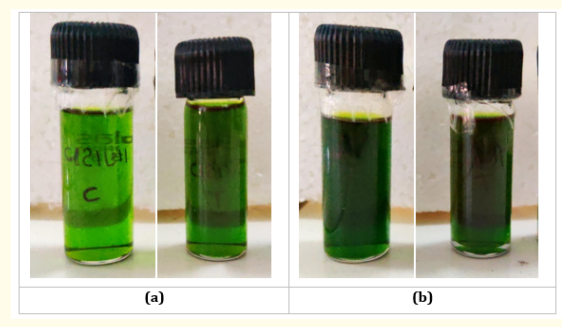

Figure 2: Plant extract in chilled acetone (Left side is Control [C], Right side is Test [T]). (a): Pisum sativum C and T. (b): Vigna angularis $\mathrm{C}$ and $\mathrm{T}$. 


\section{FTIR of plant extract}

FTIR is an essential analytical tool and it is rapid, sensitive as well as accurate [13]. The plant extracts of Pisum sativum and Vigna angularis were analysed in FTIR spectrometer by employing standard $\mathrm{KBr}$ pellet techniques. The FTIR spectrum of the above-mentioned plant extracts are shown below in figure 3 . The wavelengths are shown in table 1 [23].

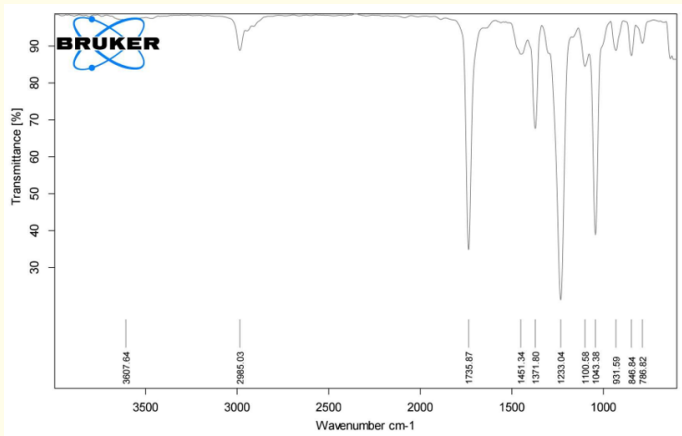

(a)

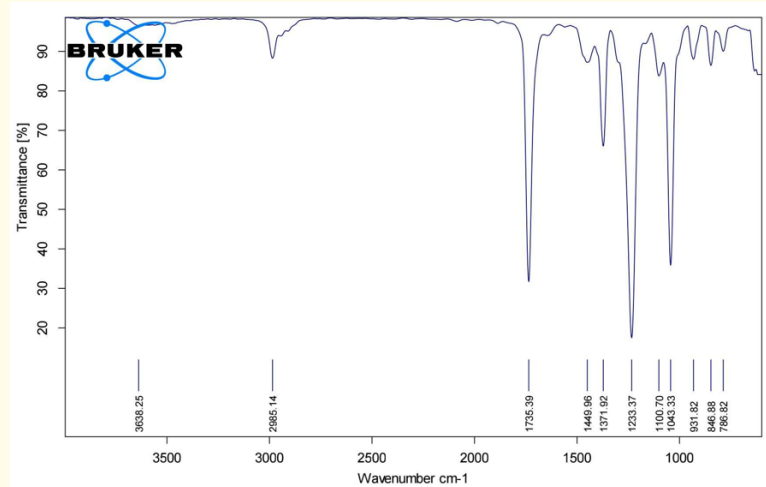

(b)

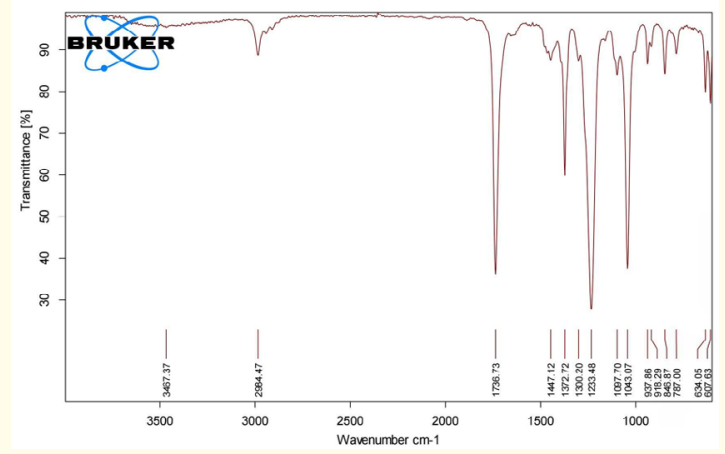

(c)

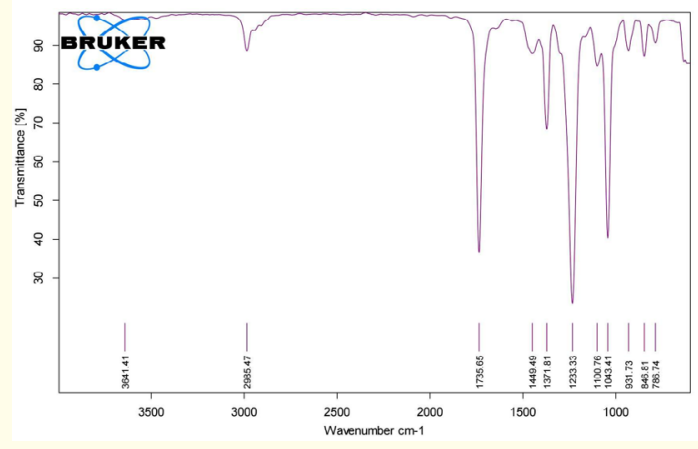

(d)

Figure 3: FTIR spectrum. (a): FTIR spectrum of Pisum sativum Control. (b): FTIR spectrum of Pisum sativum Test. (c): FTIR spectrum of Vigna angularis Control. (d) FTIR spectrum of Vigna angularis Test.

\begin{tabular}{|c|c|c|c|c|}
\hline $\begin{array}{l}\text { Functional } \\
\text { group }\end{array}$ & $\begin{array}{c}\text { Pisum } \\
\text { sativum }\end{array}$ & $\begin{array}{c}\text { Pisum } \\
\text { sativum }\end{array}$ & $\begin{array}{c}\text { Vigna } \\
\text { angularis }\end{array}$ & $\begin{array}{c}\text { Vigna } \\
\text { angularis }\end{array}$ \\
\hline & Control & Test & Control & Test \\
\hline \multirow{2}{*}{$\begin{array}{l}\text { Ester, Ketone } \\
C=0\end{array}$} & 1735.87 & 1735.39 & 1736.73 & 1735.65 \\
\hline & & & & \\
\hline $\begin{array}{l}\text { Carboxylic acid } \\
\text { O-H }\end{array}$ & 2985.03 & 2985.14 & 2984.47 & 2985.47 \\
\hline $\begin{array}{l}\text { Alcohol } \\
\mathrm{OH}\end{array}$ & 3617.64 & 3638.25 & 3467.37 & 3641.41 \\
\hline
\end{tabular}

Table 1: Wave number $\left(\mathrm{cm}^{-1}\right)$ of dominant peak obtained from plants.

From the table 1 and figure 3, it is observed that there was difference between Control and Test of Vigna angularis and Pisum sativum in alcohol wave number. In test samples, alcohol wave numbers are observed higher than the control wave number. FTIR is also possible for analysing material on the surfaces of substrate [10]. Compared to other types of characterization analysis, FTIR is quite popular. This characterization analysis is rapid, good in accuracy, and relatively sensitive [13]. FTIR is useful for determining specific functional group contained in the sample [17].

\section{Conclusion}

From this experimentation and results, characterization of consortium development from endophytic bacteria was completed 
and it was observed that the isolate D was Bacillus subtilis, isolate $\mathrm{N}$ was Bacillus tequilensis and isolate A was Bacillus amyloliquefaciens. The presence of endophytes promotes the growth of this both plants as compared with un inoculated pot studied, maximum growth observed in consortium pot in $21 \mathrm{~d}$ pot study. There was presence of alcohol group and change in wave number between two plants had been observed with control by FTIR analysis of Pisum sativum and Vigna angularis plant extract. Alcohol group $(-\mathrm{OH})$ is relevant to polyphenols present in plant extract. It might be saying that polyphenol group is changed as compared with control and test plants due to the presence of endophytes which were inoculated as biofertilizer during pot experiments.

\section{Acknowledgement}

We are thankful to Bhadaniya Nidhi, Metaliya Divya, Patel Aakruti for continuous support.

\section{Conflict of Interest}

The authors have no conflicts of interest in preparing of this research article.

\section{Bibliography}

1. Akuzawa S and Kawabata A. "Relationship among starches from different origins classified according to their physicochemical properties". Journal of Applied Glycoscience 50.2 (2003): 121-126.

2. Aseri GK., et al. "Biofertilizers improve plant growth, fruit yield, nutrition, metabolism and rhizosphere enzyme activities of pomegranate (Punica granatum L.) in Indian Thar Desert". Scientia Horticulture 117.2 (2008): 130-135.

3. Bhadaniya N., et al. "Strategy of Salt Tolerance and Productive Effects of Bacillus tequilensis Under Specific Conditions". International Journal of Scientific Engineering and Applied Science 7.4 (2021): 181-192.

4. Cousin R. "Breeding for yield and for protein content in pea" (1983).

5. Cousin R. "Peas (Pisum sativum L.)”. Field Crops Research 53.13 (1997): 111-130.

6. Cousin R., et al. "Research on genetic factors for combining pea quality (Pisum sativum)". In 1. European conference. Association Europeenne des Proteagineux (1992).

7. Cousin R., et al. "What about the genetic determinism?" Grain Legumes 1 (1993): 24-25.
8. Crosbie GB. "The relationship between starch swelling properties, paste viscosity and boiled noodle quality in wheat flours". Journal of cereal science 13.2 (1991): 145-150.

9. Dumale JV., et al. "Detection and Quantification of Auxin and Gibberellic Acid in Caulerpa racemose". International Journal of Agricultural Technology 4 (2018): 653-660.

10. Fan M., et al. "Fourier transform infrared spectroscopy for natural fibres". Fourier Transform-Materials Analysis 3 (2012): 45-68.

11. Hayakawa I and Breene WM. "A study on the relationship between cooking properties of adzuki bean and storage conditions" (1982).

12. Honda Y., et al. "Characterization of physicochemical and digestive properties of starches from various "dainagon" adzuki beans (Vigna angularis) cultivated in Japan". International Journal of Biological Macromolecules 148 (2020): 1021-1028.

13. Jaggi N and Vij DR. "Fourier transform infrared spectroscopy". In Handbook of Applied Solid State Spectroscopy. Springer, Boston, MA (2006): 411-450.

14. Jane JL., et al. "Effects of amylopectin branch chain length and amylose content on the gelatinization and pasting properties of starch". Cereal Chemistry 76.5 (1999): 629-637.

15. Jiménez-Gómez A., et al. "Increase in phenolic compounds of Coriandrum sativum L. after the application of a Bacillus halotolerans biofertilizer". Journal of the Science of Food and Agriculture 100.6 (2020): 2742-2749.

16. Juhász R and Salgó A. "Pasting behavior of amylose, amylopectin and their mixtures as determined by RVA curves and first derivatives". Starch-Stärke 60.2 (2018): 70-78.

17. Kirk RE and Othmer DF. "Encyclopedia of Chemical Technology. Pentacene to polymethine dyes". Interscience Encyclopedia, Incorporated 10 (1953).

18. Leterme P., et al. "A great intervarietal diversity for TIA in peas". Grain Legumes 1 (1993): 22-23.

19. LII CY and CHANG SM. "Characterization of red bean (Phaseolus radiatus var. Aurea) starch and its noodle quality". Journal of Food Science 46.1 (1981): 78-81.

20. Metaliya D., et al. "Various Mechanism of Bacillus subtilis as Endophyte with Ameliorate Salinity Stress". International Journal of All Research Education and Scientific Methods 9.4 (2021): 476-487. 
21. Movasaghi Z., et al. "Fourier transform infrared (FTIR) spectroscopy of biological tissues". Applied Spectroscopy Reviews 43.2 (2008): 134-179.

22. Naivikul $\mathrm{O}$ and D’Appolonia BL. "Carbohydrates of legume flours compared with wheat flour: III. Non-starchy polysaccharides". Cereal Chemistry 56 (1979): 45-9.

23. Nandiyanto ABD., et al. "How to read and interpret FTIR spectroscope of organic material”. Indonesian Journal of Science and Technology 4.1 (2019): 97-118.

24. Patel A., et al. "Augmentation of Salt Tolerance by Halotolerant Bacillus amyloliquefaciens as Highly Phosphate Solubilizer". International Journal of Creative Research Thoughts (IJCRT) 9.4 (2021): 322-330.

25. Rakesh P and Kiran P. "Identification of bacteria. Experimental Microbiology-I”. Third edition Aditya Publication, Ahmedabad, India (2004): 95-101.

26. Rathod Zalak R., et al. "Compatibility studies on different endophytes of Citrus limon antagonistic to bacterial with plant pathogen". Advances in Bioresearch 11.3 (2020): 126-129.

27. Rathod Zalak R and Saraf Meenu S. "Effect of endophytes as phosphate solubilizing bacteria on Vigna radiata plant". Vidhya a journal of Gujarat University 1.1 (2021): 59-69.

28. Rathod Z. "Developing Plant Probiotic Bacteria based Bioformulation having PGPR Properties [Masters of Philosophy thesis School of Sciences of Gujarat University of India] (2016).

29. Riaz U., et al. "Prospective roles and mechanisms of caffeic acid in counter plant stress: A mini review". Pakistan Journal of Agricultural Research 32.12 (2019): 8.

30. Sacks FM. "A literature review of Phaseolus angularis: The adsuki bean". Economic Botany 31.1 (1977): 9-15.

31. Sasaki T and Matsuki J. "Effect of wheat starch structure on swelling power". Cereal Chemistry 75.4 (1988): 525-529.

32. Sasaki T., et al. "Effect of amylose content on gelatinization, retrogradation, and pasting properties of starches from waxy and nonwaxy wheat and their F1 seeds". Cereal chemistry 77.1 (2000): 58-63.

33. Solanki MK., et al. "Co-inoculation of different antagonists can enhance the biocontrol activity against Rhizoctonia solani in tomato". Antonie Van Leeuwenhoek 112.11 (2019): 1633-1644.
34. Srichuwong S., et al. "Starches from different botanical sources II: Contribution of starch structure to swelling and pasting properties". Carbohydrate polymers 62.1 (2005): 25-34.

35. Suzuki S., et al. "The A Handbook." Korinshoin Publishers Co. Tokyo (1975).

36. Tjahjadi $\mathrm{C}$ and Breene WM. "Isolation and characterization of adzuki bean (Vigna angularis cv Takara) starch". Journal of food Science 49.2 (1984): 558-562.

37. Wani IA., et al. "Isolation, composition, and physicochemical properties of starch from legumes: A review". Starch-Stärke 68.9-10 (2016): 834-845.

38. Yooyongwech S., et al. "Arbuscular mycorrhiza improved growth performance in Macadamia tetraphylla L. grown under water deficit stress involves soluble sugar and proline accumulation". Plant Growth Regulation 69.3 (2013): 285-293.

39. Zhang LN., et al. "Consortium of plant growth-promoting rhizobacteria strains suppresses sweet pepper disease by altering the rhizosphere microbiota". Frontiers in Microbiology 10 (2019): 1668.

\section{Volume 4 Issue 12 December 2021 (C) All rights are reserved by Rathod Zalak $R$ and Saraf Meenu S.}

